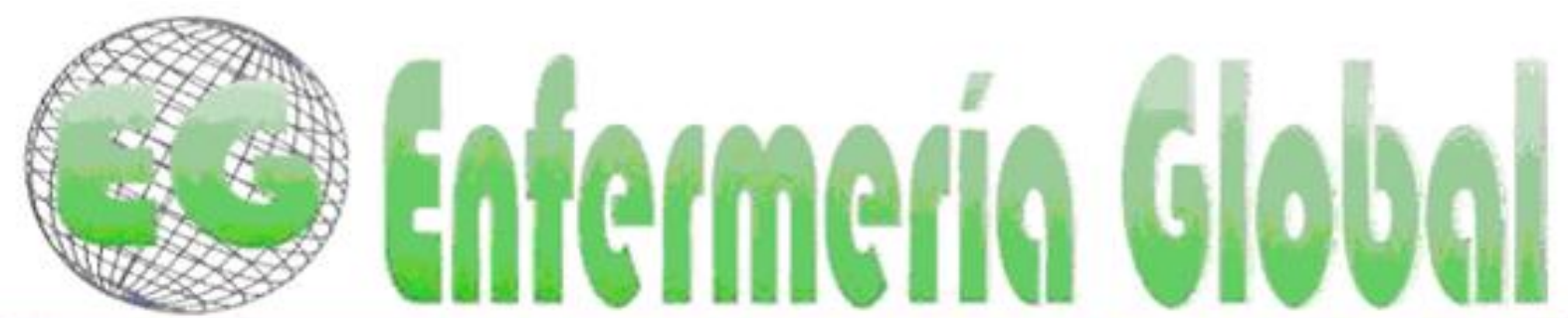

ISSN 1695-6141

Revista electrónica trimestral de Enfermería

No38

www.um.es/eglobal/

Abril 2015

\title{
CLÍNICA
}

\section{Competencia para el cuidado de la salud en mujeres con trauma raquimedular}

Competition for health care in women with spinal cord trauma

\section{*Burbano López, Margoth Consuelo}

\author{
*Especialista en Enfermería en Cuidado Crítico del Adulto; Magíster en Enfermería. Universidad del \\ Valle. Cali, Colombia. E-mail: Margot.consuelo.burbano@correounivalle.edu.co
}

Palabras Clave: mujeres; trauma raquimedular; conducta de salud; condiciones sociales

Keywords: women; spinal cord injury; health behavior; social conditions.

\section{RESUMEN}

Objetivo: Establecer la relación entre la competencia para el cuidado de la salud y algunos factores socioculturales, biológicos y la autoestima en mujeres con trauma raquimedular.

Metodología: Estudio descriptivo transversal con 44 mujeres que presentaron trauma raquimedular entre 2006 y 2010, en Cali, Colombia.

Resultados: $50 \%$ de las mujeres eran menores de 30 años; la mayoría tenían estudios de secundaria, no ejecutaban actividades laborales, pertenecían a los estratos bajos, tenían compromiso de movilidad y sensibilidad, vejiga e intestino neurogénico. La causa principal del trauma raquimedular fue herida por arma de fuego. La competencia global para el cuidado fue regular en el $43,1 \%$ de las mujeres, buena en el $40,9 \%$, excelente en el 9,1\% y deficiente en el 6,8\%. La autoestima estaba comprometida en las mujeres con vejiga e intestino neurogénico independientemente de su actividad sexual; la correlación de la competencia para el cuidado con el estrato socioeconómico y el déficit neurológico vesical fue significativa.

Conclusiones y recomendaciones: El nivel educativo alto de las mujeres puede ser una fortaleza para el cuidado. La competencia deficiente en sexualidad exige la prestación de servicios de salud sexual y reproductiva específicos para mujeres jóvenes expuestas a diferentes riesgos y relaciones de pareja insatisfactorias. Es necesario desarrollar estudios con muestras estadísticamente representativas junto con estudios cualitativos para comprender la problemática de la vida y el cuidado de la salud de las mujeres con trauma raquimedular.

\section{ABSTRACT}

Goal: To establish the relationship between the health care competency and some socio-cultural, biological factors and self esteem in women with spinal cord injury. 
Methodology: descriptive transversal study with 44 women spinal cord trauma presenting between 2006 and 2010, in Cali Colombia.

Findings: Fifty percent of women were less than 30 years old; most of them had high school education, did not work outside home, and were from low socioeconomic status, with mobility, sensibility, bladder and bowel deficits. The main cause of the injury was fire gun wound. The global competency for care was fair in $43,1 \%$ of women, good in $40,9 \%$, excellent in $9,1 \%$ and deficient in $6,8 \%$. Self esteem was compromised in women with neurogenic bladder and bowel regardless of their sexual activity. The relationship between health care competency, socio economic status, and bladder deficit was statistically significant.

Conclusions and recommendations: the women's high education level could be a positive factor for their health care. The deficiency in their competence for sexuality care calls for sexual and reproductive health services to young women exposed to several risks and unsatisfactory couple relations. It is needed to carry out studies with statistically representative samples along with qualitative studies to understand the life world and health care issues for women with spinal cord injury.

\section{INTRODUCCIÓN}

El trauma raquimedular (TRM) es una enfermedad de gente joven, en el mundo, el accidente de tránsito es responsable en más de la mitad de los casos, seguido de los accidentes laborales y deportivos. En Estados Unidos, la National Spinal Cord Injury Association ha estimado que se producen 7.800 lesiones anuales y que viven 250.000 a 400.000 personas con disfunciones medulares. La mayor tasa corresponde al grupo de edad de 16 a 34 años, la edad promedio es 33,4 años y el $82 \%$ corresponde a personas de sexo masculino ${ }^{(1)}$. En Colombia, el TRM se incrementa por causas de la violencia y en épocas de verano por el clavado en ríos y piscinas de poca profundad $^{(2)}$.

En nuestro medio, se ha evidenciado que las mujeres con TRM utilizan poco los servicios de salud generales y especializados que permiten la promoción de la salud y la prevención de complicaciones derivadas de la discapacidad por lesión neurológica. Esta situación preocupa, puesto que la literatura evidencia factores de riesgo interrelacionados entre sí que afectan la salud de las mujeres con TRM, entre los que se encuentran:

a) factores ligados a la atención sanitaria: estas mujeres reportan que a menudo se sienten aisladas, discriminadas, desalentadas y en ocasiones ignoradas y maltratadas por los equipos de salud ${ }^{(3,4)}$;

b) factores personales biológicos: existen diferencias estadísticamente significativas en cuanto al mayor riesgo que tiene la mujer con TRM de sufrir enfermedades cardiovasculares y respiratorias si comparada con el hombre que presenta la misma lesión ${ }^{(5)}$. Dado que el TRM no afecta la fertilidad en la mujer, se presentan embarazos en promedio a los 6.5 años después de la lesión y tienen en promedio 26.8 años de edad al primer embarazo ${ }^{(6)}$. Los factores de riesgo para la salud durante la gestación están relacionados con la hiperreflexia autonómica que conduce a la eclampsia y las infecciones de vías urinarias por cateterismos vesicales al riesgo de parto pre término, este en ocasiones no es detectado por la mujer debido a la pérdida de la sensibilidad $(6,7)$. Se desconoce el apoyo que estas mujeres reciben en los servicios de salud en el uso de métodos anticonceptivos.

c) factores ambientales: Algunas mujeres con TRM manifiestan sentirse infantilizadas por sus cuidadores, limitadas para tomar sus propias decisiones con relación a sí 
mismas y al medio familiar especialmente respecto a los hijos, aunque reconocen la importancia de la familia en el apoyo que ellas requieren;

d) factores ligados al estilo de vida: mantienen las relaciones con amigos(as), sin embargo, ellas mismas se alejan por temor a ser una carga, por ello permanecen más tiempo en cama y encerradas en casa ${ }^{(4,8)}$. En general, el estilo de vida de la mujer con lesión medular comparado con el del hombre difiere en cuanto al cuidado diario: mientras los hombres reciben el cuidado de las esposas y madres, las mujeres lo reciben en muchas ocasiones de un proveedor que recibe un salario ${ }^{(9)}$.

El cuidado de la salud de las personas con TRM es necesario mirarlo desde enfogues como: 1) el humanístico, según la teoría del cuidado humano de Jean Watson ${ }^{(10)}$ se puede fundamentar, entre otros principios, la esperanza, la fe y la sensibilidad para sí y para los otros. Apoyarle para que vuelva a pensar en los propios valores, mejore la autoestima y modifique la conducta de "haber perdido todo"; 2) desde los modelos de la promoción de la salud, proporcionar a las personas los medios necesarios para lograr la salud y mayor calidad de vida. Se propone abordarlo desde los patrones funcionales de Marjory Gordon ${ }^{(11)}$, que permite hacer el cuidado de la salud en lo físico, emocional, espiritual y en estrecha relación con el medio que lo rodea.

Para enfermería, como garante del cuidado integral de la salud de las personas, cobra gran interés investigar las situaciones donde se encuentren factores de riesgo como los que pueden existir en las mujeres con TRM en nuestro medio, tanto por su situación de género como por su situación de discapacidad. Por lo cual se consideró pertinente realizar un estudio para determinar la relación de la competencia personal para el cuidado de la salud con algunos factores socioculturales, biológicos y la autoestima en las mujeres con TRM.

\section{METODOLOGÍA}

\section{Diseño y muestra}

Estudio descriptivo transversal. No fue posible calcular una muestra estadísticamente representativa debido a que los estudios reportan una incidencia que incluye hombres y mujeres con una relación en algunos de 5:1 y en otros de 8:1, lo que representa una escasa población de mujeres. Igualmente, los estudios de prevalencia reportan en Norteamérica 681 a 755 por millón de habitantes en 30 años y en Europa 250 a 252 por millón de habitantes ${ }^{(12,13)}$. Calcular la muestra con estos datos representa un tamaño de muestra que requiere muchos años para realizar el estudio, por consiguiente la muestra fue determinada por conveniencia. Se tuvo en cuenta el total de mujeres que ingresaron a una institución de tercer nivel de Cali entre los años 2006 y 2010 y que atiende la mayoría de la población con TRM del Sur Occidente Colombiano. Las 44 mujeres que constituyeron la muestra representan el $96 \%$ del total de las atendidas entre estos años. Las mujeres cumplieron los siguientes criterios de inclusión: edad mayor de 18 años, clasificación del TRM Frankel A, B, C, D y E, tiempo de ocurrido el TRM mínimo de 3 meses, no tener otra patología diagnosticada. Como criterios de exclusión se consideró trastornos mentales o del estado de conciencia.

Las variables independientes fueron: factores socioculturales (estado civil, situación laboral y económica, nivel educativo, seguridad social en salud, causa de la lesión), factores biológicos (edad, nivel de la lesión, fuerza muscular, déficit sensitivo, nivel 
motor de la lesión, déficit neurológico vesical e intestinal) y autoestima; la competencia personal para el cuidado de la salud fue la variable dependiente.

\section{Instrumentos y procedimientos para recolectar los datos}

Los datos se recolectaron mediante dos cuestionarios diseñados por la investigadora: uno para medir los factores socioculturales y los factores biológicos y otro para determinar la competencia percibida de las mujeres para cuidar su salud, que se elaboró teniendo en cuenta los once patrones funcionales de Marjory Gordon ${ }^{(11)}$. La competencia se calificó a través de la escala de Likert capaz $=5$, regularmente capaz $=4$, poco capaz $=3$, incapaz $=2$ y muy incapaz $=1$, la cual permitió obtener el puntaje final para clasificar la competencia como excelente, buena, regular y deficiente. La validez de contenido de estos instrumentos se estableció a través de la evaluación de cinco expertos y por la prueba piloto.

La autoestima se midió con la Escala de Rosemberg, validada por Martín-Albo, Núñez, Navarro y Grijalvo ${ }^{(14)}$. Según estos autores la escala tiene validez de contenido y confiabilidad apropiados; sin embargo, dicha validez y confiabilidad fue realizada con estudiantes universitarios hispanoparlantes, por lo cual también se realizó una prueba piloto de la escala en mujeres con lesión medular. La información fue recolectada únicamente por la investigadora.

La validez externa del estudio puede estar disminuida porque la muestra es por conveniencia, sin embargo, el hecho de que la muestra represente el $96 \%$ de la población atendida en una institución de salud de tercer nivel en los últimos cinco años en Cali, puede contribuir a garantizar dicha validez.

Para el procesamiento y el análisis de la información se utilizó el programa estadístico SPSS versión 15. La competencia para el cuidado se analizó de manera global y por patrones para el total de la muestra y para los siguientes subgrupos: 1) mujeres con vejiga neurogénica, con intestino neurogénico y con actividad sexual, 2) mujeres con vejiga neurogénica, con intestino neurogénico y sin actividad sexual, 3) mujeres sin vejiga neurogénica, sin intestino neurogénico y sin actividad sexual, 4) mujeres sin vejiga neurogénica, sin intestino neurogénico y con actividad sexual. Para analizar las diferencias de la variable dependiente y las variables independientes se utilizaron la prueba de ANOVA y el chi-cuadrado de Pearson con $p=0.10$.

El estudio fue aprobado por el Comité Institucional de Revisión de Ética Humana de la Facultad de Salud de la Universidad del Valle mediante acta № 013-09.

\section{RESULTADOS}

\section{Factores socioculturales}

El $50 \%$ de las mujeres eran solteras, 27,3 \% convivían con una pareja; el 11,4\% se separaron de su pareja después del TRM y ninguna había conseguido pareja después de la lesión. El 27,3 \% tenía secundaria completa y el 20,5\% universitaria completa. El $70,5 \%$ de las mujeres no realizaban actividades laborales remuneradas, lo cual se refleja en que el 65,9 \% eran apoyadas económicamente por la familia; el 81,8 \% pertenecían a los estratos 1, 2 y 3 y estaban afiliadas al régimen de salud subsidiado. La principal causa de la lesión fue herida por arma de fuego en el $50 \%$ de las mujeres, seguida por caídas en el 27,3 \%. 


\section{Factores biológicos}

La edad promedio fue de 33 años con desviación estándar de 12 años y coeficiente de variación de 0,36 , es decir, la población estudiada es dispersa en cuanto a edad. La edad más frecuente fue 22 años y el $50 \%$ de la población tenía menos de 30 años. El $72,7 \%$ de las mujeres presentaba importante compromiso de la movilidad y la sensibilidad al tener clasificación Frankel A, B y C. La mayoría presentaban lesión medular a nivel torácico, vejiga neurogenica $(70,5 \%)$ e intestino neurogenico $(90,9$ $\%)$.

\section{Autoestima}

El $56,8 \%$ de las mujeres presentaron autoestima elevada, el $29,5 \%$ baja y $13,6 \%$ media; el valor promedio en la Escala de Rosemberg fue de 30,4 con un valor mínimo de 13 y máximo de 40, desviación estándar de 7,51 y coeficiente de variación de 0,25. El promedio de la autoestima fue mayor en mujeres sin vejiga ni intestino neurogénicos, independiente de si tenían o no actividad sexual, a diferencia de las mujeres con vejiga e intestino neurogénicos y actividad sexual quienes presentaron el promedio más bajo de autoestima. Según los coeficientes de variación, el nivel de autoestima fue homogéneo entre las mujeres sin vejiga e intestino neurogénicos y heterogéneo en las que no tenían estas condiciones.

\section{Competencia global para el cuidado}

La competencia global para el cuidado fue regular en el $43,2 \%$ de las mujeres, buena en el $40,9 \%$, excelente en $9,1 \%$ y deficiente en el $6,8 \%$. El valor mínimo obtenido fue 75 puntos, el máximo 224 y valor promedio 142,3 con desviación estándar de 53,6 y coeficiente de variación de 0,24 , lo que indica que hay heterogeneidad en la competencia para el cuidado entre las mujeres.

\section{Competencia para el cuidado por patrones funcionales}

Al analizar la competencia para el cuidado por patrones se encontró que era buena en los patrones manejo y percepción de la salud, metabólico nutricional, sueño y descanso.

\section{Competencia global para el cuidado de la salud según la presencia de vejiga neurogénica, intestino neurogénico y actividad sexual}

La mujeres sin vejiga e intestino neurogénico y sin actividad sexual mostraron el promedio más bajo en la competencia en contraste con las mujeres sin vejiga e intestino neurogénico y con actividad sexual que tuvieron el promedio más alto como se observa en tabla I. La competencia promedio resultó mejor entre las mujeres con vejiga e intestino neurogénicos y actividad sexual que entre las mujeres con estas condiciones y sin actividad sexual, es decir, que las mujeres con actividad sexual, independientemente del déficit vesical e intestinal, mostraron promedio más alto en la competencia para cuidarse. El nivel de competencia para el cuidado fue homogéneo únicamente entre las mujeres sin vejiga e intestino neurogénicos y sin actividad sexual (coeficiente de variación $=0,18$ ). Las diferencias de la competencia entre los grupos y en los grupos no fueron estadísticamente significativas $(F=1.404, p=$ $0,234)$. 
Tabla I: Competencia global para el cuidado de la salud de las mujeres con TRM según vejiga neurogénica, intestino neurogénico y actividad sexual. Cali.

\begin{tabular}{|l|c|c|c|c|}
\hline \multicolumn{1}{|c|}{ Grupos } & \multicolumn{3}{c|}{ Competencia para el cuidado } \\
\cline { 2 - 5 } & Escala & Media & $\begin{array}{c}\text { Desv. } \\
\text { Tip }\end{array}$ & $\begin{array}{c}\text { Coeficiente } \\
\text { de } \\
\text { variación }\end{array}$ \\
\hline $\begin{array}{l}\text { Con vejiga neurogénica, con } \\
\text { intestino neurogénico y actividad } \\
\text { sexual }\end{array}$ & Buena & 164,33 & $36,0,6$ & 0,21 \\
\hline $\begin{array}{l}\text { Con vejiga neurogénica, con } \\
\text { intestino neurogénico sin actividad } \\
\text { sexual }\end{array}$ & Buena & 138,90 & 30,34 & 0,21 \\
\hline $\begin{array}{l}\text { Sin vejiga, sin intestino } \\
\text { neurogénico y con actividad } \\
\text { sexual }\end{array}$ & Buena & 184 & 35,35 & 0,42 \\
\hline $\begin{array}{l}\text { Sin vejiga neurogénica, sin } \\
\text { intestino neurogénico y sin } \\
\text { actividad sexual }\end{array}$ & Regular & 114,50 & 21,20 & 0.18 \\
\hline
\end{tabular}

Relación entre la competencia global para el cuidado en los factores socioculturales, biológicos y la autoestima

Se encontró diferencias estadísticamente significativas en la correlación de la competencia global para el cuidado según el estrato social $(p=0,021)$ y con el déficit neurológico vesical $(p=0,087)$. No se encontró correlación significativa con los demás factores Tabla II.

Tabla II: Competencia para el cuidado según factores socioculturales y biológicos de las mujeres con TRM, Cali.

\begin{tabular}{|c|c|c|c|}
\hline Factores & Correlación & $\begin{array}{l}\text { F de } \\
\text { ANOVA }\end{array}$ & $\mathbf{p}$ \\
\hline \multirow{7}{*}{$\begin{array}{c}\text { Factores } \\
\text { socioculturales }\end{array}$} & Competencia vs estado civil & 1,221 & 0,318 \\
\hline & Competencia vs trabajo actual & 1,278 & 0,290 \\
\hline & $\begin{array}{l}\text { Competencia vs ingresos } \\
\text { económicos }\end{array}$ & 0,788 & 0,508 \\
\hline & Competencia vs estrato social & 3,054 & 0,021 \\
\hline & Competencia vs nivel educativo & 1,404 & 0,234 \\
\hline & Competencia vs seguridad social & 1,716 & 0,192 \\
\hline & $\begin{array}{l}\text { Competencia vs causa de la } \\
\text { lesión }\end{array}$ & 0,132 & 0,877 \\
\hline \multirow[t]{5}{*}{ Factores biológicos } & Competencia vs edad & 1,818 & 0,185 \\
\hline & $\begin{array}{l}\text { Competencia vs clasificación } \\
\text { Frankel }\end{array}$ & 0,706 & 0,592 \\
\hline & $\begin{array}{l}\text { Competencia I vs nivel de la } \\
\text { lesión }\end{array}$ & 0,376 & 0,695 \\
\hline & Competencia vs fuerza muscular & 0,890 & 0,497 \\
\hline & $\begin{array}{l}\text { Competencia vs alteración a la } \\
\text { sensibilidad al dolor }\end{array}$ & 1,203 & 0,279 \\
\hline
\end{tabular}




\begin{tabular}{|l|c|c|}
\hline $\begin{array}{l}\text { Competencia vs déficit } \\
\text { neurológico vesical }\end{array}$ & $\mathbf{3 , 0 7 9}$ & $\mathbf{0 , 0 8 7}$ \\
\hline $\begin{array}{l}\text { Competencia vs déficit } \\
\text { neurológico intestinal }\end{array}$ & 0,004 & 0,952 \\
\hline
\end{tabular}

No obstante al analizar el nivel de la competencia global para el cuidado agrupada en dos categorías: deficiente-regular y buena-excelente, se encontraron hallazgos importantes desde el punto de vista clínico. El nivel de competencia fue deficienteregular en las mujeres con los siguientes factores socioculturales: sin pareja, sin trabajo, que dependen económicamente de su familia, pertenecen al estrato bajo, con estudios de primaria y secundaria, afiliadas al régimen subsidiado de salud y con lesión por arma de fuego; con factores biológicos como lesión medular a nivel cervical aún sin alteración de la función sensitiva o motora (Frankel E) y menores de 30 años. De igual manera fue deficiente-regular en mujeres con autoestima baja con significancia estadística $(p=0,05)$. Tabla III.

Tabla III: Nivel de competencia para el cuidado según factores biológicos y autoestima de las mujeres con TRM, Cali.

\begin{tabular}{|c|c|c|c|c|}
\hline \multirow{2}{*}{$\begin{array}{l}\text { Factores } \\
\text { biológicos }\end{array}$} & \multicolumn{2}{|c|}{ Competencia } & \multirow[t]{2}{*}{$\mathrm{X}^{2}$} & \multirow[t]{2}{*}{$\mathbf{P}$} \\
\hline & $\begin{array}{l}\text { Deficiente - } \\
\text { Regular }\end{array}$ & $\begin{array}{l}\text { Bueno - } \\
\text { excelente }\end{array}$ & & \\
\hline $\begin{array}{l}\text { Frankel: } \\
\text { - A, B,C } \\
\text { - } \mathrm{D} \\
\text { - } \mathrm{E}\end{array}$ & $\begin{array}{l}16 \\
6 \\
1\end{array}$ & $\begin{array}{l}16 \\
5 \\
0\end{array}$ & 2.362 & 0.66 \\
\hline $\begin{array}{l}\text { Nivel de la lesión: } \\
\text { - Cervical } \\
\text { - Torácico } \\
\text { - Lumbar }\end{array}$ & $\begin{array}{c}6 \\
13 \\
4\end{array}$ & $\begin{array}{c}3 \\
12 \\
6\end{array}$ & 1.352 & 0.50 \\
\hline $\begin{aligned} \text { Fuerza muscular: } & \\
- & 0-3 \\
- & 4 \\
- & 5\end{aligned}$ & $\begin{array}{l}15 \\
6 \\
2\end{array}$ & $\begin{array}{l}16 \\
5 \\
0\end{array}$ & 6.690 & 0.24 \\
\hline $\begin{array}{l}\text { Alteración de la } \\
\text { sensibilidad: } \\
\text { - Si } \\
\text { - No }\end{array}$ & $\begin{array}{c}3 \\
20\end{array}$ & $\begin{array}{c}3 \\
18\end{array}$ & 0,14 & 0.90 \\
\hline $\begin{array}{c}\text { Déficit vesical: } \\
\text { - Si } \\
\text { - No }\end{array}$ & $\begin{array}{c}15 \\
8\end{array}$ & $\begin{array}{c}16 \\
5\end{array}$ & 0,635 & 0.42 \\
\hline $\begin{array}{l}\text { Déficit intestinal: } \\
\text { - Si } \\
\text { - No }\end{array}$ & $\begin{array}{c}3 \\
20\end{array}$ & $\begin{array}{c}3 \\
18\end{array}$ & 2.27 & 0,13 \\
\hline $\begin{array}{l}\text { Edad: } \\
\qquad \begin{aligned} \bullet & <\text { de } 30 \\
& \text { años } \\
& >\text { de } 30 \\
& \text { años }\end{aligned}\end{array}$ & $\begin{array}{c}14 \\
9\end{array}$ & $\begin{array}{c}8 \\
13\end{array}$ & 2.27 & 0,13 \\
\hline
\end{tabular}




\begin{tabular}{|c|c|c|c|c|}
\hline Autoestima: & 11 & 3 & 5.715 & 0.05 \\
- Baja & 7 & 11 & & \\
- Media & 5 & 7 & & \\
- Alta & 5 & 7 & \\
\hline
\end{tabular}

\section{DISCUSIÓN}

Los factores socioculturales de las mujeres del estudio, excepto el nivel educativo, pueden considerarse factores que afectan la competencia para el cuidado de la salud. La mayoría no tenía pareja que las apoyara o estimulara a cuidarse, dependían económicamente de sus familias, pertenecían a los estratos socioeconómicos bajos y estaban afiliadas al régimen subsidiado de salud, que no provee los recursos para el cuidado de la salud como son las sondas de cateterismo vesical intermitente, medicamentos para el tratamiento del dolor, la infección urinaria y úlceras por presión. Sumado a la condición socioeconómica que generaba dificultades para transportarse a las instituciones de salud. Estas situaciones son paradójicas, si se tiene en cuenta que la Constitución Política de Colombia, Artículos 47, 54 y 68 establece los derechos de las personas en situación de discapacidad, enmarcados en el concepto del apoyo social de carácter multidimensional que incluye el acceso a los servicios de salud con la provisión de los recursos necesarios para promoción de la salud y la rehabilitación ${ }^{(15)}$. Blanes L, Carmagnani MIS, Ferreira LM ${ }^{(16)}$ reportan riesgos en la calidad de vida y la salud independientemente del género en personas con TRM cuando estas personas dependen de la seguridad social proporcionada por el Estado, es decir, cuando las personas son de estratos socioeconómicos bajos, tienen nivel educativo deficiente y son jóvenes con menor oportunidad de empleo.

Richards E, Tepper M, Whipple B, Komisaruk B ${ }^{(17)}$ y Sing y Sharma ${ }^{(18)}$ encontraron que entre el $8 \%$ y el $48 \%$ de las mujeres que conviven con una pareja se separaron a consecuencia de la adaptación que requiere la pareja a la nueva vida sexual, a los cambios en las relaciones familiares y al rol del cónyuge o compañero. Existe poca evidencia científica sobre el ajuste conyugal de las mujeres con lesión medular, además los servicios de salud no contemplan este aspecto de la vida de las mujeres como relevante y por consiguiente no proveen servicios de orientación al respecto, lo cual podría producir en las mujeres sentimientos de frustración al no poder resolver la crisis de pareja que afrontan. Cuando las mujeres logran estos apoyos, experimentan satisfacción con la vida y mejores resultados en la competencia personal y la auto motivación ${ }^{(17)}$.

Las mujeres del presente estudio tenían un nivel educativo superior al encontrado en estudios de Richards y colaboradores ${ }^{(17)}$ y Sing y Sharma ${ }^{(18)}$ que reportan $5 \%$ y $12 \%$ para los niveles secundario y universitario y $22 \%$ sin educación. Por lo tanto, el nivel educativo de estas mujeres puede ser un factor que favorece su competencia para el cuidado, permite al personal de salud utilizar varias estrategias de comunicación para mejorar la calidad de vida e independencia de estas mujeres. En este sentido, se ha encontrado que las personas con TRM por medio de Telesalud pueden acceder a los servicios de salud, obtener información sobre sus derechos y tener intercambio con otros pacientes sobre el cuidado de la salud ${ }^{\left({ }^{(19)}\right.}$. Igualmente, se espera que las mujeres con más educación formal tengan mayor capacidad para comprender la situación y encontrar recursos para resolverla. 
Para Shackelford, Farley y Vines ${ }^{(9)}$, existen diferencias en los factores de riesgo para el TRM entre hombres y mujeres, en especial porque los hombres están expuestos a factores de violencia o conductas de riesgo como la conducción de automóviles a mayor velocidad y al uso de sustancias psicoactivas. Estos autores reportaron la colisión vehicular como la causa del TRM en el $50 \%$ tanto de hombres como en mujeres y la violencia como la segunda causa sólo en hombres. El hallazgo del presente estudio evidencia un cambio en esta tendencia, pues la lesión por arma de fuego seguida por la colisión vehicular fueron las principales causas del TRM de las mujeres. Según Van de Ven, Post, Witte, Van Den y Heuvel ${ }^{(19)}$ las personas con TRM por violencia tienen mayor riesgo de depresión y estrés postraumático hasta varios años después de la lesión. También Krausea Broderickb L, Broylesb ${ }^{(20)}$ encontraron que este riesgo es mayor en las mujeres que en los hombres, especialmente porque las mujeres son más susceptibles a presentar poca adaptabilidad a la situación de discapacidad.

La influencia de los factores de riesgo biológicos como la edad, compromiso de la movilidad, sensibilidad y déficit neurológico e intestinal en la competencia para el cuidado son similares a los de otros autores. Al ser estas mujeres jóvenes y en edad productiva y reproductiva, la planificación y desarrollo de los servicios y estrategias de intervención para la promoción de la salud debe estar acorde con este hecho. La población joven con TRM es considerada de alto riesgo por la pérdida de la identidad, la frustración por no poder hacer lo mismo que sus pares y el consecuente riesgo de refugiarse en el cigarrillo, el alcohol y el consumo de sustancias psicoactivas ${ }^{(9,21,22 \text {, }}$ ${ }^{23)}$. En este estudio no se encontró riesgos en ninguna de las mujeres con relación al uso de alcohol o sustancias psicoactivas, sin embargo, esto solo se exploró con una pregunta.

Se considera que las conductas generadoras de salud en personas con TRM están determinadas por los patrones establecidos antes de la lesión ${ }^{(19)}$, sin embargo, el compromiso de la movilidad y de la sensibilidad son factores intervinientes en la motivación de estas personas para la realización de cualquier actividad física. El $72,7 \%$ de las mujeres del estudio presentaron Frankel A, B y C por lo tanto, estas mujeres son más proclives a no participar en actividades recreativas y deportivas, como también por las barreras emocionales derivadas de haber sido víctimas de la violencia. La actividad física y el ejercicio son muy importantes en las mujeres con TRM por el mayor riesgo de osteoporosis debida a la menopausia prematura que padecen ${ }^{(7,24)}$.

Las complicaciones por vejiga neurogénica son responsables de reingresos hospitalarios de las personas con TRM ${ }^{(25,26,27)}$. El 70,5\% de las mujeres del estudio por la dificultad en el acceso al meato urinario, no realizan el cateterismo vesical con la frecuencia requerida, prefieren el uso de pañal y eliminan por rebosamiento. Esto es causa de complicaciones como infección urinaria, sepsis, reflujo retrógrado hacia uréteres por aumento de la presión vesical y falla renal. La urostomía y otros catéteres ureterocutáneos son considerados como la opción para el cuidado de la vejiga neurogénica en las mujeres con TRM cuadripléjicas; sin embargo, ningún régimen de salud en Colombia los considera y se requieren más estudios para evaluar su beneficio ${ }^{(28)}$.

Para el $70,4 \%$ de las mujeres estudiadas, la autoestima podría considerarse un factor protector para su salud; no obstante, casi la tercera parte tenían baja autoestima y fue clínicamente relevante en las mujeres con vejiga e intestino neurogénicos y actividad 
sexual. Samuel, Marini y $\mathrm{Ku}(29,30,31)$ establecen una relación lineal entre vejiga neurogénica y sentimientos de insatisfacción o depresión, incluso 3,8 veces mayor en mujeres que en hombres. Otros estudios relacionan la baja autoestima de las personas con TRM, con la pérdida del empleo, aspectos ambientales del hogar y públicos que no proveen condiciones para la movilización en sillas de ruedas, problemas asociados al deterioro del cuerpo como el dolor, la espasticidad, infecciones urinarias y las úlceras por presión, varios de estos factores socioculturales y biológicos relacionados con las mujeres de este estudio ${ }^{(16)}$.

El $50 \%$ de las mujeres con competencia regular y deficiente para el cuidado de la salud presentó igual calificación en los patrones rol relaciones, enfrentamiento al estrés, sexualidad-reproducción y eliminación. Esto significa que el asesoramiento inter pares, la familia y los amigos son cruciales en el cuidado de estas mujeres, a través de ellos logran desarrollar sentimientos de esperanza y motivación en el marco del rol y las relaciones personales. Existe la tendencia a considerar estas mujeres asexuales; los estudios demuestran que para las mujeres con TRM, la sexualidad es el núcleo de lo que piensan y sienten de su cuerpo, las relaciones sexuales satisfactorias aumentan la confianza para el cuidado de la vejiga, intestino neurogénico y logran control sobre decisiones con relación a sus vidas. Se debe tener en cuenta la posibilidad del embarazo para la remisión a los servicios de salud que brindan orientación al respecto, e incluir el seguimiento al trastorno menstrual, donde se presentan períodos de amenorrea seguidos de otros con abundante sangrado y complicaciones como la anemia. Hay que estimular a la mujer para que realice del auto examen de mama, y considerar en la rehabilitación la mamografía como la citología en salud preventiva. Se debe establecer medidas terapéuticas para los riesgos de la menopausia prematura como es la osteoporosis ${ }^{(7)}$.

Se recomienda realizar estudios con muestras mayores y en diferentes regiones del país para tener un conocimiento científico más amplio de la situación de las mujeres con TRM en Colombia que conduzca a plantear una política nacional del cuidado de la salud de estas mujeres que contemple servicios específicos y coherentes con las necesidades y problemas de ellas y los derechos reconocidos por el Estado en la Constitución de 1991. También, desarrollar estudios, preferiblemente cualitativos, que profundicen en los aspectos psicológicos como la autoestima y la sexualidad de las mujeres con TRM para comprender la trascendencia de estos aspectos en su vida y cuidado de la salud.

\section{REFERENCIAS BIBLIOGRÁFICAS}

1. Neira J, Di Stéfano C. Trauma raquimedualar. En: Cuidados Intensivos Neurológicos. Santiago de Chile: Mediterráneo Ltda; 2004.

2. Montoya A. Trauma raquimedular agudo. En: Pedroza A. Compendio de Neurocirugía. Bogotá: Quebor; 2002.

3. Castañeda I. Reflexiones teóricas sobre las diferencias de salud atribuibles a género. Rev. Cubana Salud Pública 2007; 33(2): 1-20.

4. Álvarez R, Sugiyama E. La experiencia de vivir con una lesión traumática: desde el análisis de la narrativa. PCS 2004; 6(2): 22-33.

5. Denton M, Prusb S, Walters V. Gender differences in Health: a Canadian study of the psiychosocial, structural and behavioural determinants of health. Rev. Socsimed 2004; 58: 2585-2600.

6. Gay Pasley Earl RN. Autonomic Dysreflexia: A Special Challenge for the Nursing Care of the Woman with Quadriplegia during Childbirth. MCN 2002; 27 (2). 
7. Cesario, SK. Nurses Can Help Affected Women \& Their Families Achieve Pregnancy, Birth. AWHONN Lifelines 2002; 6(3): 226-232. [Internet]. Disponible en: www.google.com. Consultado 2 de mayo del 2009.

8. Shackelford M, Farley T, Vines Ch. A comparison of women and men with spinal cord injury. Spinal Cord 1998; 36: 337-339.

9. Watson J. Filosofia y Ciencia del Cuidado. En: Marrinier A y Raile M. Modelos y Teorías en Enfermería. España: Liderdúples; 2007. p. 91-115.

10. Gordon M. Diagnóstico de Enfermería: Proceso y Aplicación. 3aㅗ ed. St. Louis: Ed. Mosby-Year Book; 1994.

11. Wyndaele M, Wyndaele JJ. Incidence, prevalence and epidemiology of spinal cord injury: what learns a worldwide literature survey? Spinal Cord 2006; 44: 523-529.

12. Dahlberg A, Kotila M, Leppa $M$, Kautiainen $H$, Alaranta $H$. Prevalence of spinal cord injury in Helsinki. Spinal Cord 2005; 43: 47-5

13. Albo M, Núñez, JL, Navarro JG, Grijalvo F. The Rosenberg Self-Esteem Scale: Translation and Validation in University Students. The Spanish Journal of Psychology 2007; 10(2): 458-467.

14. República de Colombia, Presidencia de la República, Red de Solidaridad Social, Ministerio de Protección Social, Comité Regional de Rehabilitación de Antioquia. Política Pública de Discapacidad: fortalecimiento y movilización social. Medellín: Begón Ltda. Medellín; [en internet] 2005. p. 39 - 56. [Consultado 2008 junio].Disponible en: www.google.com.

15. Blanes L, Carmagnani MIS, Ferreira LM. Quality of life and self-esteem of persons with paraplegia living in Sao Paulo Brazil. Qual Life Res 2009; 18:15-21.

16. Richards E, Tepper M, Whipple B, Komisaruk B. Women with Complete Spinal Cord Injury: A Phenomenological Study of Sexuality and Relationship Experiences. Journal of Sex \& Marital Therapy 2001; 27: 615-23.

17. Sing R, Sharma S. Sexuality and Women with Spinal Cord Injury. Sexuality and Disability 2005; 23 (1): $21-33$.

18. Van de Ven L, Post M, Witte L, Van Den Heuvel W. Strategies for autonomy used by people with cervical spinal cord injury: A qualitative study. Disability and Rehabilitation 2008; 30(4): 249-26.

19. Krausea J, Broderickb L, Broylesb J. Subjective well-being among AfricanAmericans with spinal cord injury: An exploratory study between men and women. Neuro Rehabi 2004; 19: 81-89.

20. Kreuter M. Spinal cord injury and partner relationships. Spinal Cord 2000; 38: 2-6.

21. Kennedy P, Duff J. Post traumatic stress disorder and spinal cord injuries. Spinal Cord 2001; 39: 1-10.

22. Galvin R, Godfrey P. The impact of coping on emotional adjustment to spinal cord injury (SCI): review of the literature and application of a stress appraisal and coping formulation. Spinal Cord 2001; 39: 615-627.

23. Pentland W, Walker J, Minnes P, Tremblay M, Brouwer B, Gould M. Women with spinal cord injury and the impact of aging. Spinal Cord 2002; 40: 374-387.

24. Fonte Nancy. Urological Care of the Spinal Cord-Injured Patient. JWOCN 2008; 35(3): 323-331.

25. Dryden DM, Et al. Utilización de los servicios de salud posterior a la lesión espinal: un seguimiento de seis años. Spinal Cord 2004; 42: 513-525.

26. Middleton JW, Lim K, Taylor L, Soden R, Rutkowski S. Patterns of morbidity and rehospitalization following spinal cord injury. Spinal Cord 2004; 42: 359-367.

27. Dahlberg A, Perttila I, Wuokko E, Ala-Opas M. Bladder management in persons with spinal cord lesion. Spinal Cord 2004; 42: 694-698.

28. Samuel VW, Moses J, North N, Smith H, Thorne K. Spinal cord injury rehabilitation: the experience of women. Spinal Cord 2007; 45: 758-764. 
29. Marini I, Rogers L. Self- esteem differences among persons with spinal cord injury. Rehabilitation Counseling Bulletin 1995; 38 (3): 198.

$30 . \mathrm{Ku} \mathrm{JH}$. The management of neurogenic bladder and quality of life in spinal cord injury. Journal compilation 2006; 98: 739- 745.

Recibido: 30 de agosto 2013; Aceptado: 23 de octubre 2013 